\title{
Occupation Date Range
}

National Cancer Institute

\section{Source}

National Cancer Institute. Occupation Date Range. NCI Thesaurus. Code C93607.

The date and time span specifying the start and end of a job. 